Gut, 1979, 20, 886-891

\title{
Trypsinogen variants in pancreatic juice of healthy volunteers, chronic alcoholics, and patients with pancreatitis and cancer of the pancreas
}

\author{
H. RINDERKNECHT, I. G. RENNER, AND C. CARMACK \\ From the Veterans Administration Hospital, Sepulveda, and University of California-San Fernando \\ Valley Medical Program, Los Angeles, California, and the \\ University of Southern California School of Medicine, Los Angeles, California, USA
}

\begin{abstract}
SUMmary Polyacrylamide gel electrophoresis of pure pancreatic juice from 14 healthy normal subjects, 11 chronic alcoholics without detectable pancreatic disease, 15 patients with pancreatitis, and two with cancer of the pancreas consistently demonstrated the presence of two variants of trypsinogen with different electrophoretic mobilities. In healthy normal subjects the proportion of cationic to anionic trypsinogen was invariably greater than 1 and averaged about 2 . In chronic alcoholics, patients with pancreatitis or cancer of the pancreas, this ratio, with a single exception, was below one and averaged about $0 \cdot 45$. The extraordinary consistency of these findings suggests that the quantitative relationship between cationic and anionic trypsinogen in human pancreatic juice may be a very sensitive indicator of incipient or existing pancreatic pathology. The most acceptable explanation for the reversal of the normal zymogen ratio in pancreatic disease is a selective increase in the synthesis of the anionic variant relative to that of the cationic species. Total trypsinogen concentrations differed widely from one another in the three patient groups, but the ratio of cationic to anionic trypsinogen exhibited little change and remained below 1 . Our results also demonstrate for the first time a specific effect of chronic alcohol abuse on the secretory profile of a pancreatic enzyme in human subjects. A newly discovered minor, trypsinogen-like component of human pancreatic juice was found to be significantly increased in pancreatic juice of chronic alcoholics, decreased in pancreatic secretions of patients with pancreatitis, and barely detectable in those of two patients with cancer of the pancreas.
\end{abstract}

Trypsinogen of mammalian species including man (Rinderknecht and Geokas, 1973) occurs in two variants differing slightly from one another in their biochemical properties such as isoelectric point, susceptibility to inhibitors, substrate specificity, and so on. Robinson et al. (1972) recently analysed pure pancreatic juice (PPJ) from 34 patients free of pancreatic disease and found that pancreatic secretions of different subjects contained either both or only one of the two trypsinogen variants. The authors suggested that the occurrence of two species of trypsinogen at different frequencies in different segments of the population may represent a genetically determined polymorphism of this zymogen. In view of the important function of trypsin as activator of

Received for publication 26 April 1979 pancreatic zymogens and its putative role in the pathogenesis of pancreatitis and pancreatic malignancy (White and Allan, 1974), we decided to investigate the possibility of a correlation between the frequency pattern of trypsinogen variants and the incidence of pancreatic disease. The work presented here describes the distribution of trypsinogen variants in PPJ of normal, healthy subjects, chronic alcoholics, patients with pancreatitis, and gives a preliminary account of the trypsinogen variants in PPJ of two patients with cancer of the pancreas. Alcoholics were included in this investigation because of the possibility that alcohol abuse, one of the main aetiological factors in pancreatitis and possibly cancer of the pancreas (Burch and Ansari, 1968; Dani and Nogueira, 1976), might result in biochemical abnormalities in pancreatic secretions detectable before the appearance of clinical symptoms. 


\section{Methods}

\section{SUBJECTS}

\section{Normal subjects}

Fourteen fasted, healthy volunteers (nine males and five females aged 19-71 years) were investigated. Seven of these subjects were total abstainers, the others were light social drinkers consuming alcoholic beverages in moderate amounts at irregular intervals.

\section{Chronic alcoholics}

These patients were selected from the detoxification ward of LAC-USC Medical Center. All admitted to heavy alcohol intake for 12 to 30 years. There was no historical, clinical, or biochemical evidence of pancreatic disease in any of these patients. None of them exhibited any features of advanced liver disease, spider naevi, ascites, peripheral oedema, or hepatic coma, but clinical examination revealed moderate hepatomegaly in these patients. None of them was subjected to pancreatography.

\section{Patients with pancreatitis}

Fourteen patients in this group were chronic alcoholics. One patient denied any history of alcohol abuse, but had experienced multiple episodes of abdominal pain for several years. Diagnosis of alcoholic pancreatitis was established in 10 patients on the basis of admitted chronic alcohol abuse, periodic episodes of abdominal pain, raised urinary and serum amylase, and an abnormal pancreatogram obtained by endoscopic retrograde pancreatography (ERP). Five of these patients had minimal chronic pancreatitis and five had moderate to advanced chronic pancreatitis. Minimal chronic pancreatitis was indicated by abnormalities in the pancreatogram which included features of irregularity, constriction, dilatation, ectasia, and pooling of contrast medium in the minor duct system involving primary, secondary, and tertiary branches. Pancreatograms in patients with moderate or advanced chronic pancreatitis showed the above abnormalities in addition to strictures and dilatation of the main pancreatic duct. In two additional patients with similar histories, the pancreatograms were normal, but the clinical features of chronic relapsing pancreatitis were present and raised amylase to creatinine clearance ratios were obtained. One patient who was not subjected to ERP showed evidence of severe pancreatic calcification on abdominal radiography and one patient who did not have an ERP had raised urine and serum amylase and a raised amylase to creatinine clearance ratio. The only patient with presumed idiopathic chronic relapsing pancreatitis who denied alcohol abuse furnished a pancreatogram showing changes associated with moderate chronic pancreatitis.
Patients with cancer of pancreas

Some 30 patients with cancer of the pancreas were subjected to ERP. Although this procedure was successful diagnostically in most cases, only two patients furnished satisfactory secretory profiles. Obstruction of the pancreatic duct by the tumour prevented collection of PPJ in some patients or pancreatic tissue no longer responded to stimulation by hormones in others. Cancer of the pancreas in one patient was diagnosed at the time of collection of PPJ by a grossly abnormal pancreatogram showing a stricture at the junction of body and tail of the pancreas. In the other patient who had a normal pancreatogram at the time of collection of PPJ, cancer of the pancreas was diagnosed at necropsy by histology.

\section{COLLECTION PROCEDURE}

Pure pancreatic juice was obtained by cannulation of the main pancreatic duct as described by Rinderknecht et al. (1978b). Secretions were collected at one minute intervals for a total of 20 minutes after initial stimulation with secretin and cholecystokininpancreozymin (CCK-PZ) 10 minutes later. Samples were assayed immediately or stored at $-70^{\circ} \mathrm{C}$. The procedure was explained in detail to volunteers and patients and informed consent obtained from each individual. The protocol was approved by the VA Human Studies Subcommittee and LAC-USC Medical Center Human Research Committee.

\section{ASSAYS}

Trypsinogen present in each of the one minute collections of PPJ was determined as active trypsin after activation with human enteropeptidase as described previously (Rinderknecht et al, 1978b). Trypsin activity is expressed in $\mu \mathrm{g}$ active site-titrated bovine trypsin/ml PPJ. One $\mu \mathrm{g}$ active-site titrated trypsin equals $70 \mathrm{mIU}$. Trypsin (Worthington Biochemical Corp., Freehold, N J) is about $55-60 \%$ active. Pancreatic trypsin inhibitor was determined according to Rinderknecht et al. (1978b).

\section{ELECTROPHORESIS}

Gels containing $8.5 \%$ polyacrylamide and measuring $10.5 \mathrm{~cm}$ were prepared in siliconised tubes $0.7 \mathrm{~cm}$ in diameter. Acrylamide was dissolved in buffer containing $0.2 \mathrm{~mol} / 1$ glycine, $0.05 \mathrm{~mol} / 1 \mathrm{Tris}$, and 0.001 mol/l EDTA and the solution was adjusted to pH 8.2. After polymerisation with tetramethylethylenediamine and ammonium persulphate, excess persulphate was removed by pre-electrophoresis. Pure pancreatic juice $(50-100 \mu l$ of the one minute collection showing the highest concentration of trypsinogen after CCK-PZ administration) was placed on the gel and subjected to electrophoresis at 
Table 1 Mean peak concentrations of trypsinogens and zymogen-x in PPI after CCK-PZ stimulation; comparison between normal subjects, chronic alcoholics, patients with pancreatitis, and patients with cancer of the pancreas

\begin{tabular}{|c|c|c|c|c|c|c|}
\hline & \multicolumn{4}{|c|}{ Mean peak concentrations and range } & \multicolumn{2}{|c|}{ Comparison $\mathrm{P}$ by student's $t$ test } \\
\hline & $\begin{array}{l}\text { Normal } \\
\text { subjects } \\
N=14\end{array}$ & $\begin{array}{l}\text { Chronic } \\
\text { alcoholics } \\
N=11\end{array}$ & $\begin{array}{l}\text { Patients with } \\
\text { pancreatitis } \\
N=15\end{array}$ & $\begin{array}{l}\text { Patients with } \\
\text { cancer of } \\
\text { pancreas } \\
N=2\end{array}$ & $\begin{array}{l}\text { Alcoholics } \\
\text { vs. } \\
\text { normals }\end{array}$ & $\begin{array}{l}\text { Pancreatitics } \\
\text { vs. } \\
\text { normals }\end{array}$ \\
\hline $\begin{array}{l}\text { Total } \\
\text { trypsinogen* }\end{array}$ & $\begin{array}{l}324 \\
(86-903)\end{array}$ & $\begin{array}{l}1017 \\
(513-2788)\end{array}$ & $\begin{array}{l}311 \\
(52-972)\end{array}$ & $97 ; 135$ & $<0.001 \uparrow$ & NS \\
\hline $\begin{array}{l}\text { Cationic } \\
\text { trypsinogen* }\end{array}$ & $\begin{array}{l}187 \\
(60-599)\end{array}$ & $\begin{array}{l}299 \\
(108-809)\end{array}$ & $\begin{array}{l}77 \\
(6-288)\end{array}$ & $11 ; 35$ & NS & $0.05 \downarrow$ \\
\hline $\begin{array}{l}\text { Anionic } \\
\text { trypsinogen }\end{array}$ & $\begin{array}{l}92 \\
(17-340)\end{array}$ & $\begin{array}{l}615 \\
(316-1729)\end{array}$ & $\begin{array}{l}176 \\
(54-768)\end{array}$ & $83 ; 97$ & $<0.001 \uparrow$ & NS \\
\hline $\begin{array}{l}\text { Ratio: cationic/ } \\
\text { anionic trypsinogen }\end{array}$ & $\begin{array}{l}2.0 \\
(1.1-9)\end{array}$ & $\begin{array}{l}0.49 \\
(0.23-0.89)\end{array}$ & $\begin{array}{l}0.45 \\
(0.22-1.34)\end{array}$ & $0.13 ; 0.36$ & $<0.001 \downarrow$ & $<0.001 \downarrow$ \\
\hline Zymogen-X $X^{*}$ & $\begin{array}{l}34 \\
(9-108)\end{array}$ & $\begin{array}{l}87 \\
(34-223)\end{array}$ & $\begin{array}{l}11 \\
(0.1-39)\end{array}$ & $3 ; 1$ & $<0.01 \uparrow$ & $<0.025 \downarrow$ \\
\hline
\end{tabular}

*Expressed as $\mu \mathrm{g}$ active site-titrated bovine trysin/ml PPJ.

$5^{\circ} \mathrm{C}$ and constant current ( $5 \mathrm{mAmp} /$ tube) for five to six hours. The gels were removed and segmented into $0.5 \mathrm{~cm}$ sections. Each segment was homogenised in an ice water bath with $1 \mathrm{ml} 0.05 \mathrm{M}$ Tris maleate buffer, pH 5.4 containing $4 \mathrm{mmol} / 1 \mathrm{CaCl}_{2}$ and $3.5 \mathrm{mmol} / 1$ glycodeoxycholic acid. The supernatant $(50 \mu l)$ of each homogenate was assayed for active trypsin. Experiments showing the presence of active trypsin were rejected. Each supernatant containing only unactivated zymogens was then activated by addition of $100 \mu$ l human enteropeptidase solution $(11 \mu \mathrm{IU} / \mathrm{ml}$; Rinderknecht et al., 1976). After standing at $7^{\circ} \mathrm{C}$ overnight $10 \mu \mathrm{l}$ of the supernatant of each homogenate was assayed for trypsin. Three peaks with trypsin activity were observed in each sequence of homogenates. The tubes located between the first and third peak were re-assayed in the presence of 2 KIU (Kallikrein inhibitor unit) of Trasylol (FBA Pharmaceuticals, Division of Baychem Corp., New York, NY). 1 KIU inhibits $150 \mathrm{ng}$ active-site titrated bovine trypsin.

\section{STATISTICS}

The skewness of the data obtained in each of the series of subjects investigated necessitated transformation to log scale for comparison by Student's $t$ test. The means given in Table 1 have been converted back to antilog of the results obtained in the calculations.

\section{Results}

Figure 1 represents a typical profile of the trypsinogen variants obtained by gel electrophoresis of PPJ from normal healthy subjects. It shows the presence of two anionic forms of the zymogen with different electrophoretic mobilities. To avoid confusion, the less anionic variant, which predominated in all normal subjects, is henceforth termed 'cationic' and the more anionic variant 'anionic' trypsinogen. The Figure also shows an additional minor component with trypsinogen-like properties. As an active enzyme (enzyme-X) it is, however, not inhibited by human or Kunitz bovine pancreatic trypsin inhibitor as shown on the right hand side of Fig. 1 (Rinderknecht et al. 1978a). Figure 2A illustrates a typical profile of the two trypsinogens in PPJ of chronic alcoholics. It can be seen that, in these patients, in contrast with normal subjects, the predominant zymogen is the anionic variant. Profiles of patients with pancreatitis or cancer of the pancreas also exhibited a preponderance of anionic trypsinogen (Fig. 2, B and C).

The reproducibility of such profiles was found to be quite satisfactory, particularly in view of the

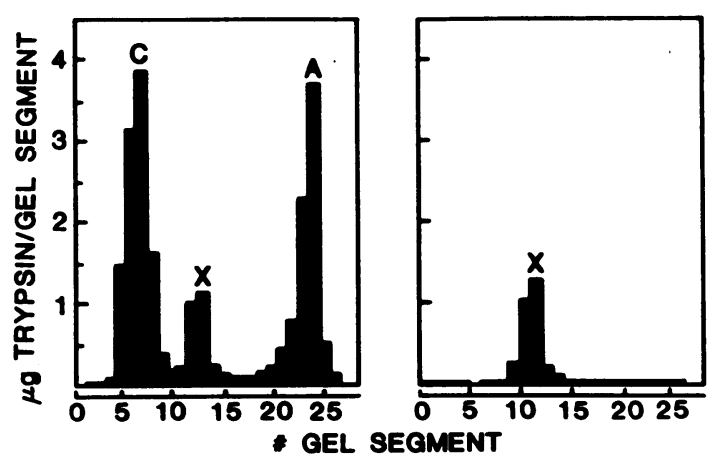

Fig. 1 Electrophoretic profile of trypsinogen variants in $P P J$ from a normal, healthy subject. PPJ applied $50 \mu l$. Zymogens measured as active site-titrated bovine trypsin as described in the section on Methods. Left: $C=$ cationic trypsinogen, $X=$ zymogen of enzyme- $X$, $A=$ anionic trypsinogen. Right: activity determined in the presence of 2 KIU Trasylol. 
Table 2 Ratio of cationic/anionic trypsinogen at different points during CCK-PZ stimulated collection of PPI

\begin{tabular}{lll}
\hline Normal subjects & $\begin{array}{l}\text { Time of collection } \\
(\text { min })\end{array}$ & $\begin{array}{l}\text { Ratio of cationic/anionic } \\
\text { trypsinogen }\end{array}$ \\
\cline { 2 - 3 } FF & 18 & 1.02 \\
& 19 & 1.36 \\
BB & 20 & 1.55 \\
& 14 & 1.40 \\
& 16 & 1.30 \\
Pancreatitis & 23 & 1.20 \\
CC & 12 & 0.89 \\
& 13 & 0.92 \\
PC & 14 & 0.69 \\
& 18 & 0.52 \\
Pancreatic cancer & 19 & 0.50 \\
AS & 14 & $0.10 ; 0.16$ \\
& 15 & $0.28 ; 0.28$ \\
& 10 & 0.23 \\
\hline
\end{tabular}

complexity of the experimental procedure that was used. Thus, repeat electrophoreses performed on PPJ from normal individuals or patients gave the following results expressed as ratios of cationic to anionic trypsinogen: normal subject no. 1: 2.4, 2.3, 3.0 ; normal subject no. $2: 1.3,1.8$; patient with pancreatitis: $0.6,0.6,0.5$. The recovery of total trypsinogen applied (including zymogen-X) for all normal subjects and patients was $103 \pm 36 \%$ (SD). Low concentrations of chymotrypsin measured by a fluorometric method (Rinderknecht and Fleming, 1975) were detected occasionally as contaminant of part of the cationic trypsin peak, but they had no effect on the trypsin determinations. No chymotrypsin was found in the anionic trypsin or enzyme-X peak of the electrophoretic profile.
Human pancreatic juice contains two trypsin inhibitors. The larger, less anionic component was found to be located, at least partially, below the cationic trypsinogen peak, the smaller variant in the area of enzyme-X. Because the relative proportions of the two inhibitors could not be measured for each individual and are capable of inhibiting only a minor part of the total potential trypsin activity, they were omitted from consideration in the following calculations.

Table 1 presents a comparison of the data obtained by electrophoresis of PPJ from a series of 14 normal healthy subjects, 11 chronic alcoholics, 15 patients with pancreatitis, and two patients with cancer of the pancreas. Trypsinogen as well as enzyme- $X$ are expressed as active site-titrated bovine trypsin $/ \mathrm{ml}$ PPJ. The results indicate striking differences between normal subjects and the three groups of patients studied. In chronic alcoholics, concentrations of total trypsinogen, anionic trypsinogen, and enzyme$X$ zymogen were found to be two to six times higher than those observed in normal individuals. Cationic trypsinogen, however, showed only a slight increase which was not statistically significant, similar to that found previously in alcoholics for chymotrypsinogen proelastase, and trypsin inhibitor (Renner et al., 1978). The increase in anionic trypsinogen in patients with pancreatitis was not statistically significant, in contrast with that found in alcoholics. On the other hand, cationic trypsinogen in patients with pancreatitis showed a statistically significant decrease. In the patients with cancer of the pancreas the concentration of anionic trypsinogen was within the normal range, but that of cationic trypsinogen greatly below normal or barely detectable. The remarkable change in the distribution of the tryp-
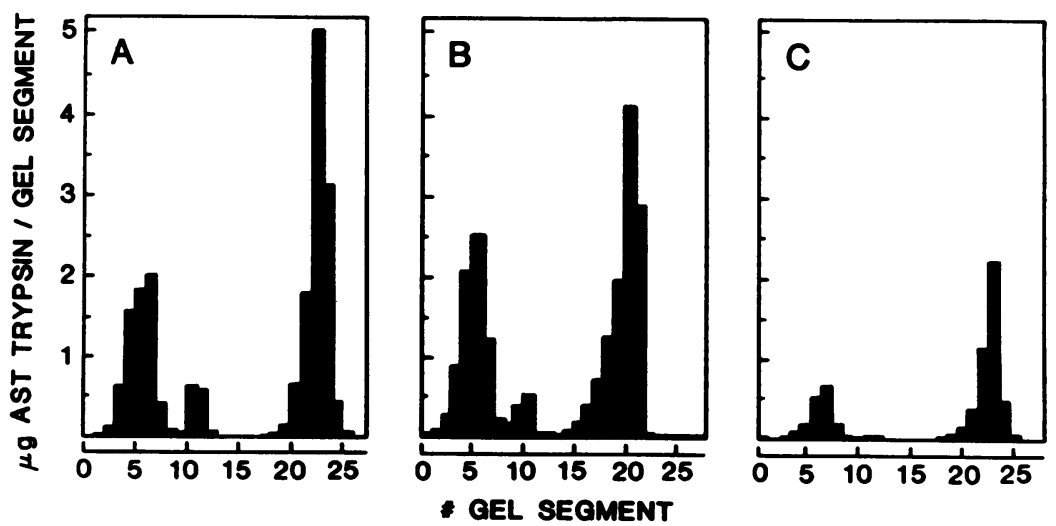

Fig. 2 Electrophoretic profiles of trypsinogen variants in PPJ from: $A=$ chronic alcoholic (PPJ applied $20 \mu \mathrm{l}), B=$ patient with chronic alcoholic pancreatitis (PPJ applied $100 \mu \mathrm{l}), C=$ patient with cancer of the pancreas (PPJ applied $100 \mu \mathrm{l})$. 
sinogen variants in the patient groups is particularly striking when expressed as the ratio of cationic to anionic trypsinogen. This ratio without exception was above 1 in normal subjects and below 1 in chronic alcoholics. All but one of the pancreatitis patients showed a ratio below 1 . The patients with cancer of the pancreas exhibited particularly low ratios, as seen in Table 1 .

The mean concentration of the minor component zymogen-X discovered by us in PPJ (Rinderknecht et al., 1978a) was higher than normal in chronic alcoholics, but below normal in patients with pancreatitis and extremely low in patients with cancer of the pancreas. These differences were statistically significant.

\section{Discussion}

The results of our investigation clearly demonstrate the presence of two trypsinogen variants in PPJ of every individual tested and thus are at variance with the concept of a genetic polymorphism of this zymogen with an uneven distribution in the population as postulated by Robinson et al. (1972). The discrepancy in these findings may be due to differences in methodology and in the type of pancreatic secretion used for investigation. Whereas Robinson and co-workers used basal PPJ, we studied secretions obtained at peak stimulation with cholecystokininpancreozymin.

In normal non-alcoholic individuals cationic trypsinogen invariably was the more abundant of the two variants. It constituted about $65 \%$ of the total trypsin activity as measured by our method. The same proportion of this zymogen in human PPJ has been reported recently by Guy et al. (1978). However, our findings indicate that the ratio of cationic to anionic trypsinogen, normally close to 2 , is very labile and susceptible to reversal to a value of less than 1 by as yet undefined biochemical changes in pancreatic tissue. Such changes apparently result from the prolonged action of ethanol and probably other noxious agents or conditions associated with pancreatitis and cancer of the pancreas. Table 1 shows that the change of this ratio in chronic alcoholics is attributable to a highly significant, selective increase (about six-fold over normal) of anionic over cationic trypsinogen and not to a decrease of the cationic variant. In the group of pancreatitis patients with a mean ratio: cationic to anionic trypsinogen of 0.45 , anionic trypsinogen also showed an increase above the normal level, but this finding lacked statistical significance. Cationic trypsinogen on the other hand was significantly decreased in this group. It must be emphasised that these results reflect a trend in a grossly heterogeneous group of patients. Classification of the patients into groups according to the stage of their disease might, in a much larger study, refine the data to correspond more accurately with the clinical condition of the patients. In both patients with cancer of the pancreas, the anionic species of trypsinogen remained in the low normal range and the decrease in the ratio: cationic to anionic trypsinogen to less than 1 was due exclusively to a reduction of the cationic variant.

The mechanism involved in the increased secretion of anionic vs. cationic trypsinogen in PPJ of our patient groups is unknown. The high recovery rate of trypsinogen applied in the electrophoretic analyses certainly seems to preclude selective destruction of the cationic variant in our procedure. Elsewhere (Renner et al., 1978), we reported that the mean flow rate of pancreatic juice after sequential stimulation with secretin and cholecystokinin-pancreozymin was similar for normal subjects and chronic alcoholics - that is, $3.26 \mathrm{ml} v s .3 .31 \mathrm{ml} / \mathrm{min}$-and that the concentrations of chymotrypsinogen, proelastase, as well as trypsin inhibitor, were increased slightly in alcoholics without, however, reaching statistical significance. The increase in trypsinogen concentration, on the other hand, was highly significant as illustrated also in Table 1 . Therefore, the most acceptable explanation for our findings is selective stimulation of the gene coding for anionic trypsinogen and increased synthesis of this species compared with that of the cationic variant in chronic alcoholics. The preponderance of anionic over cationic trypsinogen in PPJ of patients with cancer of the pancreas or pancreatitis could be explained on the same basis. Whereas the concentration of cationic trypsinogen (Table 1), chymotrypsinogen, proelastase and trypsin inhibitor in our patients with cancer of the pancreas was below normal (unpublished results) reflecting reduced secretory capacity of the pancreas, the level of anionic trypsinogen remained in the normal range. The heterogeneous group of patients with pancreatitis, most of whom were alcoholics, exhibited enzyme profiles varying between those of chronic alcoholics without pancreatic disease and those of the cancer patients. Although increased release of anionic trypsinogen due to greater sensitivity to CCK-PZ in the patient groups must be considered also as a potential factor in this mechanism, the concept of a faster release of one variant relative to the other is not in accord with the data given in Table 2. However, the accuracy of the complex procedure for the determination of the ratio of zymogens may well not be high enough to detect the increase in ratio with time that would be expected from such a mechanism. Our observations suggest that detection of a ratio of cationic/anionic tryp- 
sinogen of less than 1 in PPJ may be a sensitive indicator of incipient or established pancreatic pathology. Investigations of the effect of biliary disease, as well as various medications thought to be associated with the development of pancreatitis, are in progress and should shed additional light on this hypothesis. Determination of the minimum level of alcohol intake required for induction of anionic trypsinogen will also be of great interest.

The potential role and significance of increased concentrations of anionic trypsinogen in the pathogenesis of pancreatic disease is open to speculation. Louvard and Puigserver (1974) recently reported that the anionic form of bovine and porcine trypsinogen is activated more readily by enteropeptidase than the cationic variant, but this does not necessarily pertain to other enzymes which might be more likely to be involved in intrapancreatic activation of trypsinogen than enteropeptidase. However, should this analogy be valid, it would seem plausible that greatly raised concentrations of anionic trypsinogen with an increased susceptibility to activation in the presence of only normal (Renner et al., 1978) or even decreased levels of inhibitor might severely compromise an important defence mechanism against premature activation of zymogens in the pancreas of subjects thus affected. Reduced concentrations of trypsin inhibitor observed by us in some patients with pancreatitis and cancer of the pancreas (unpublished results) tend to support this view.

It is of interest here to note that the secretion of the zymogen of enzyme- $X$ was increased significantly in chronic alcoholics, but decreased markedly in patients with pancreatitis (Table 1) or cancer of the pancreas. The identity of this enzyme has not been fully extablished (Rinderknecht et al., 1978a) and clarification of its role in pancreatic physiology and pathophysiology must await further research.

This work was supported by the Medical Research Service of the Veterans Administration and the National Cancer Institute, Grant No. CA 20222-2 through the National Pancreatic Cancer Project, and a grant from the LAC-USC Comprehensive Cancer Center. The authors thank Dr. Gocka, Veterans Administration Hospital Sepulveda, for his assistance with statistical evaluations, Ronnie Friedman and Peggy Koyama for their excellent technical assistance, and the volunteers and patients for their cooperation and dedication.

\section{References}

Burch, G. E., and Ansari, A. (1968). Chronic alcoholism and carcinoma of the pancreas. A correlative hypothesis. Archives of Internal Medicine, 122, 273-275.

Dani, R., and Nogueira, C. E. D. (1976). Chronische Kalzifizierende Pancreatitis in Brasilien - eine Analyse von 92 Fällen. Chronic calcifying pancreatitis in Brazil. Analysis of 92 cases. Leber Magen Darm, 6, 272-275.

Guy, O., Lombardo, D., Bartelt, D. C., Amic, J., and Figarella, C. (1978). Two human trypsinogens. Purification, molecular properties and N-terminal sequences. Biochemistry, (Washington), 17, 1669-1675.

Louvard, M. N., and Puigserver, A. (1974). On bovine and porcine anionic trypsinogens. Biochimica et Biophysica Acta, 371, 177-185.

Renner, I. G., Rinderknecht, H., Valenzuela, J. E., and Douglas, A. P. (1978). Abnormalities in pure pancreatic juice from human alcoholics. Clinical Research, 26, $112 \mathrm{~A}$.

Rinderknecht, H., and Fleming, R. M. (1975). A new highly sensitive and specific assay for chymotrypsin. Clinica Chimica Acta, 59, 139-146.

Rinderknecht, H. and Geokas, M. C. (1973). Anionic and cationic trypsinogens (trypsins) in mammalian pancreas. Enzyme, 14, 116-130.

Rinderknecht, H., Renner, I. G., and Carmack, C. (1976). Activation of human pancreatic juice. Clinica Chimica Acta, 73, 369-372.

Rinderknecht, H., Renner, I. G., Carmack, C., Friedman, R., and Koyama, P. (1978a). A new protease in human pancreatic juice. Clinical Research, 26, 112 A.

Rinderknecht, H., Renner, I. G., Douglas, A. P., and Adham, N. F. (1978b). Profiles or pure pancreatic secretions obtained by direct pancreatic duct cannulation in normal healthy human subjects. Gastroenterology, 75, 1083-1089.

Robinson, L. A., Kim, W. J., White, T. T., and Hadorn, B. (1972). Trypsins in human pancreatic juice - their distribution as found in 34 specimens. Scandinavian Journal of Gastroenterology, 7, 43-45.

White, T. T., and Allan, B. J. (1974). Intrapancreatic activation of proteases in the etiology of pancreatitis and cancer of the pancreas. Medical Clinics of North America, 58, 1305-1310. 